\title{
Factores de riesgo asociados con embarazos no deseados en mujeres estudiantes de medicina
}

\author{
Diana Patricia Vanegas-Coveña, ${ }^{\text {* }}$ Tesifón Parrón-Carreño, ${ }^{2}$ Cayetano Aranda-Torres ${ }^{2}$ y \\ Raquel Alarcón-Rodríguez ${ }^{2}$

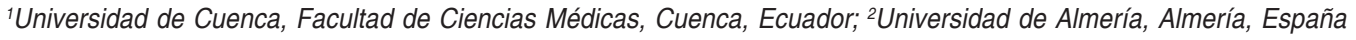

\section{Resumen}

Introducción: La mayoría de los embarazos en adolescentes y adultos jóvenes son involuntarios y muchos son consecuencia del uso inconsistente de los métodos anticonceptivos. Objetivo: Analizar los factores de riesgo que pueden influir en las adolescentes que presentan embarazos no deseados. Método: Estudio descriptivo transversal en estudiantes de medicina de Ecuador, para el cual se utilizó la Encuesta Nacional de Salud Sexual y Reproductiva 2013, escala APGAR familia y escala socioeconómica de Graffar-Méndez Castellanos. Resultados: Existió diferencia estadísticamente significativa en la edad de inicio de la vida sexual activa (18.11 \pm 1.45$)$ de las estudiantes que se embarazaron en comparación con las que no se embarazaron (19.22 \pm 2.28). La edad promedio al embarazo fue de $20.41 \pm 2.18$ ); no usaron protección en su primera relación sexual, $59.3 \%$ de las que tuvieron embarazo y $32 \%$ de las que no lo tuvieron. El embarazo fue más frecuente en las residentes de ciudades (100\%), católicas (85.2\%), pertenecientes a estrato socioeconómico medio alto (55.6 \%) y a familias con disfunción moderada (40.7\%). Conclusiones: Un porcentaje importante de estudiantes de medicina tuvo algún embarazo no deseado a temprana edad; la mayoría procedía de familias con disfunción moderada y de un nivel socioeconómico medio alto.

PALABRAS CLAVE: Estudiantes de medicina. Embarazo. Anticoncepción. Funcionalidad familiar. Estrato socioeconómico.

\begin{abstract}
Introduction: Most pregnancies in adolescents and young adults are unwanted and many are the consequence of inconsistent contraception use. Objective: To analyze the risk factors that may influence on female adolescents with unwanted pregnancies. Method: Cross-sectional, descriptive study in Ecuadorian female medical students, where the 2013 National Sexual and Reproductive Health Survey, the family APGAR scale, and the Graffar-Méndez Castellanos socio-economic scale were used. Results: There was statistically significant difference in the age of active sexual life initiation between those who became pregnant (18.11 \pm 1.45$)$ and those who did not (19.22 \pm 2.28 ). Average age at pregnancy was $20.41 \pm 2.18$; $59.3 \%$ of those who had a pregnancy and $32 \%$ of those without pregnancy did not use protection in their first intercourse. Pregnancy was more common in city residents (100\%), Catholic females (85.2\%), who belonged to middle-high (55.6\%) and middle-high socioeconomic strata (29.6\%) and to families with moderate dysfunction (40.7\%). Conclusions: $A$ significant percentage of adolescents in our study had an unwanted pregnancy at an early age despite being young undergraduate medical students, coming from moderately dysfunctional families and belonging to a middle-high socioeconomic status.
\end{abstract}

KEY WORDS: Medical students. Pregnancy. Contraception. Family functionality. Socioeconomic stratum.

Correspondencia:

*Diana Patricia Vanegas-Coveña

E-mail: diana.vanegas@ucuenca.edu.ec
Fecha de recepción: 01-09-2018

Fecha de aceptación: 19-03-2019

DOI: 10.24875/GMM.19004627
Gac Med Mex. 2019;155:357-362

Disponible en PubMed www.gacetamedicademexico.com 


\section{Introducción}

La sexualidad es un concepto amplio que abarca intereses, comportamientos, funcionamiento, satisfacción, relaciones íntimas y autopercepción de la sexualidad. Históricamente ha sido considerada en un contexto biomédico, con énfasis en el ciclo de respuesta sexual, comportamientos heteronormativos y heterosexistas. Una visión integral debe incorporar los aspectos emocional, social e intelectual. Por el contrario, las experiencias somáticas representan diversas experiencias sexuales y se centran en el placer. ${ }^{1}$

Entre adultos se habla poco de sexualidad adolescente, ya que tocar el tema se considera un detonante para desencadenar actos sexuales. Se mantiene como un tabú, reproducido a través del silencio, mitos y emociones negativas; consecuentemente, la posibilidad de su abordaje abierto e integral desde un enfoque de derechos es baja, lo que impacta negativamente en la prevención del embarazo adolescente. ${ }^{2}$

Estudios sobre los comportamientos sexuales en estudiantes de la Escuela Superior Politécnica del Chimborazo, Ecuador, encontraron que $46.24 \%$ de las mujeres inició su vida sexual activa a la edad promedio de 18 años; $52.33 \%$ no utilizó métodos anticonceptivos durante su primera relación. ${ }^{3}$

En un estudio en Cuenca, Ecuador, se consideró que el inicio de las relaciones sexuales y la posibilidad de un embarazo precoz impedían alcanzar la independencia socioeconómica, en una sociedad en la que un embarazo temprano está vinculado con el ciclo de la pobreza. ${ }^{4,5}$

Los partos de jóvenes entre 15 y 19 años representan $11 \%$ de los nacimientos en el mundo, aproximadamente 16 millones cada año. ${ }^{6}$ Las adolescentes tienen tasas desproporcionadamente altas de morbilidad derivadas de su conducta sexual, incluyendo infecciones de transmisión sexual y embarazos no deseados.?

En Portugal se observó $14.7 \%$ de embarazos adolescentes. ${ }^{8}$

En países subdesarrollados, $43 \%$ de embarazos son no planificados y son más prevalentes en mujeres pobres, del ámbito rural y con bajo nivel educativo. Cada año se producen 89 millones de embarazos no planificados y 48 millones de abortos. ${ }^{9,10}$ En un estudio en Colombia, el embarazo adolescente se relacionó con la pobreza, el bajo nivel educativo y la deserción escolar, persistiendo como determinantes estructurales la clase social, la desigualdad económica y social. ${ }^{11}$ En Francia y Gran Bretaña, los encuestados de grupos socioeconómicos más altos tenían menos probabilidades de embarazo antes de los 20 años. ${ }^{12}$

En América Latina y el Caribe, el embarazo en adolescentes es una prioridad de salud pública ya que ocupa el segundo lugar en el mundo, después de África subsahariana. Ecuador es el tercer país de la región con la tasa más alta de embarazo en adolescentes, después de Nicaragua y República Dominicana. ${ }^{13,14}$

En los últimos años, particularmente en Ecuador, ha cambiado la percepción del embarazo, transformación de la familia y concepción de la adolescencia como grupo social, lo que incide en el embarazo en esta etapa de la vida y perpetúa el círculo de violencia, pobreza y procesos educativos interrumpidos. ${ }^{15}$

La mayoría de los embarazos en adolescentes y adultos jóvenes son involuntarios y muchos son consecuencia del uso inconsistente de anticoncepción. ${ }^{6}$

Numerosas adolescentes conciben sus proyectos de vida dentro del matrimonio y la maternidad; en esos casos, el embarazo se puede experimentar más como un escape o solución que como un problema. ${ }^{2}$

La funcionalidad familiar, factores culturales, biológicos, religiosos, emocionales y ambientales influyen en las decisiones sexuales de los adolescentes, lo que subraya la importancia de la familia en la salud sexual y reproductiva en todo el mundo. ${ }^{16}$ Freud manifestaba: "la infancia es el destino", de nuestras primeras relaciones interpersonales dependerá nuestra relación con el mundo. En una familia funcional, los conflictos son vividos como diferencia de opiniones entre los miembros y no amenazan la estabilidad familiar; en la disfuncional, el conflicto se percibe como reto a la autoridad y como riesgo de desestabilización del sistema, por lo que se evita o se reprime..$^{17}$ En Colombia se encontró la disfuncionalidad familiar como factor relacionado con la maternidad temprana:10,18 resultados similares se obtuvieron en Ecuador en $2013^{19} \mathrm{y}$ en México, con $50.8 \%$ de embarazos no deseados en adolescentes provenientes de familias disfuncionales. ${ }^{20}$

El número de universitarios que utilizan el preservativo en su primera relación sexual es bajo y desciende conforme avanzan en su vida sexual. ${ }^{21}$

En el proyecto CERCA, de Cuenca, Ecuador, se observó que la mayoría de los adolescentes sexualmente activos no usan sistemáticamente anticonceptivos. ${ }^{22}$ Otro estudio en Cuenca registró que $90 \%$ de adolescentes conocía los métodos anticonceptivos, sin embargo, 70 \% declaró que su utilización no era frecuente. ${ }^{23} \mathrm{~A}$ pesar del uso inicial de algún método anticonceptivo, $51.9 \%$ de las adolescentes reportó haber estado alguna vez embarazada. ${ }^{24}$ 
Entre estudiantes de los Países Bajos, la prevalencia del uso de preservativo en la última relación sexual fue de $68.2 \%$ y de píldora, de $65.2 \% .^{25}$

Sobre la base de los trabajo mencionados, en los que se ha encontrado un alto número de embarazos no deseados como una problemática social que requiere atención urgente y en los que se han asociado relaciones familiares disfuncionales y niveles de pobreza, se llevó a cabo este estudio para analizar los posibles factores que pueden influir en los embarazos no deseados en estudiantes de las universidades de Cuenca.

\section{Objetivo}

El objetivo principal del estudio fue analizar los factores de riesgo que pueden influir en las adolescentes con embarazos no deseados.

\section{Método}

Se realizó un estudio descriptivo transversal en mujeres estudiantes del internado (año de prácticas preprofesionales) de medicina de todas las universidades del Azuay, Ecuador (Universidad de Cuenca, Universidad Católica de Cuenca, Universidad del Azuay).

La población de estudio estuvo formada por 127 mujeres matriculadas durante el curso académico 2016-2017 en todas las universidades del Azuay y que habían iniciado su vida sexual activa. Las que aún no habían mantenido relaciones sexuales fueron excluidas del estudio. Para la recolección de datos se utilizó la Encuesta Nacional de Salud Sexual y Reproductiva 2013, del Ministerio de Salud de Argentina, para mujeres. También se recolectó información sociodemográfica (edad, residencia, religión) y variables relacionadas con las relaciones sexuales (edad de inicio, voluntariedad de la relación, uso de métodos anticonceptivos, embarazo no deseado).

Para establecer el grado de funcionalidad familiar se utilizó la escala APGAR familiar, que muestra cómo perciben los miembros de la familia el nivel de funcionamiento de la unidad en forma global. ${ }^{26}$ Esta herramienta fue diseñada en 1978 por el doctor Gabriel Smilkstein (Washington), quien la propuso como un instrumento para los equipos de atención primaria, en su aproximación al análisis de la función familiar. EI APGAR familiar evalúa cinco funciones básicas de la familia (adaptación, participación, gradiente de recurso personal, afecto y recursos). ${ }^{27}$

Esta escala fue validada por Bellon et al. en 1996. Su consistencia interna fue buena, con alfa de
Cronbach de $0.770 .{ }^{28}$ El grado de funcionalidad familiar se interpreta de la siguiente forma:

- Normal, 17 a 20 puntos.

- Disfunción leve, 16 a 13 puntos.

- Disfunción moderada, 12 a 10 puntos.

- Disfunción severa, $\leq 9$ puntos.

La escala utilizada para medir el nivel socioeconómico fue la de Graffar-Méndez Castellanos, con cuatro dimensiones: profesión del jefe de la familia, instrucción de la madre, fuente de ingreso y condiciones de alojamiento. Fue validada en la Universidad Autónoma de México con alfa de Cronbach (0.69 de confiabilidad). ${ }^{29,30}$ Cada variable tiene cinco categorías con puntuaciones progresivas. Un marcador final clasifica a los sujetos en cinco categorías: ${ }^{: 1,32}$

- Nivel socioeconómico I alto, 4 a 6 puntos.

- Nivel socioeconómico II medio alto, 7 a 9 puntos.

- Nivel socioeconómico III medio, 10 a 12 puntos.

- Nivel socioeconómico IV medio bajo, 13 a 16 puntos.

- Nivel socio económico $\mathrm{V}$ bajo, 17 a 20 puntos.

Para el manejo estadístico se utilizó el programa estadístico SPSS versión 22. Se efectuó análisis univariante: para las variables cualitativas se calcularon frecuencias con sus respectivos porcentajes y para cuantitativas, media \pm desviación estándar.

Para la comparación de las variables cualitativas se utilizó chi cuadrada, para una significación estadística de $p<0.05$; para la comparación de variables cuantitativas, la $U$ de Mann-Whitney.

\section{Resultados}

Las participantes fueron 127 mujeres universitarias, con un promedio de edad de $24 \pm 1.86$ años en el momento del estudio; $21.2 \%$ había tenido un embarazo no deseado (END).

En la Tabla 1 se describen las características sociodemográficas de las participantes, en función de si habían tenido o no un END. Eran católicas $85 \%$ de las mujeres que habían tenido un END y $90 \%$ de las que no. Todas las mujeres que habían tenido un END y $94 \%$ de aquellas que no lo habían tenido, residían en el sector urbano.

Los resultados de la escala socioeconómica de Graffar Méndez Castellanos mostraron que 55.6 \% de las mujeres con END pertenecía a estrato socioeconómico medio alto y $29.6 \%$ al medio; al nivel medio bajo correspondió $14.8 \%$. No hubo mujeres de los estratos alto ni bajo con END. El $58 \%$ de las mujeres que no habían tenido ningún embarazo pertenecía a 
Tabla 1. Comparación de las características sociodemográficas de las mujeres que han tenido o no embarazo

\begin{tabular}{|c|c|c|c|c|c|}
\hline \multirow[t]{3}{*}{ Variables } & \multicolumn{4}{|c|}{ Embarazo } & \multirow[t]{3}{*}{$p$} \\
\hline & \multicolumn{2}{|c|}{ Sí } & \multicolumn{2}{|c|}{ No } & \\
\hline & \multicolumn{2}{|c|}{ Media \pm DE } & \multicolumn{2}{|c|}{ Media \pm DE } & \\
\hline \multirow[t]{2}{*}{ Edad } & 27 & $24.96 \pm 3.03$ & 100 & $23.75 \pm 1.31$ & $<0.01^{*}$ \\
\hline & $\mathrm{n}$ & $\%$ & $n$ & $\%$ & \\
\hline $\begin{array}{l}\text { Religión } \\
\text { Católica } \\
\text { No católica } \\
\text { Ateísmo }\end{array}$ & $\begin{array}{c}23 \\
3 \\
1\end{array}$ & $\begin{array}{l}85.2 \\
11.1 \\
3.7\end{array}$ & $\begin{array}{c}90 \\
9 \\
1\end{array}$ & $\begin{array}{c}90 \\
9 \\
1\end{array}$ & $>0.05^{\star}$ \\
\hline $\begin{array}{l}\text { Residencia } \\
\text { Urbana } \\
\text { Rural }\end{array}$ & $\begin{array}{c}27 \\
0\end{array}$ & $\begin{array}{c}100 \\
0\end{array}$ & $\begin{array}{c}94 \\
6\end{array}$ & $\begin{array}{c}94 \\
6\end{array}$ & $>0.05^{\star}$ \\
\hline $\begin{array}{l}\text { Estrato socioeconómico } \\
\text { I Alto } \\
\text { II Medio alto } \\
\text { III Medio } \\
\text { IV Medio bajo } \\
\text { V Bajo }\end{array}$ & $\begin{array}{c}0 \\
15 \\
8 \\
4 \\
0\end{array}$ & $\begin{array}{c}0 \\
55.6 \\
29.6 \\
14.8 \\
0\end{array}$ & $\begin{array}{c}0 \\
58 \\
28 \\
14 \\
0\end{array}$ & $\begin{array}{c}0 \\
58 \\
28 \\
14 \\
0\end{array}$ & $>0.05^{\star}$ \\
\hline $\begin{array}{l}\text { Funcionalidad familiar } \\
\text { Normal } \\
\text { Disfunción leve } \\
\text { Disfunción moderada } \\
\text { Disfunción severa }\end{array}$ & $\begin{array}{c}3 \\
6 \\
11 \\
7\end{array}$ & $\begin{array}{l}11.1 \\
22.2 \\
40.7 \\
25.9\end{array}$ & $\begin{array}{l}17 \\
25 \\
42 \\
16\end{array}$ & $\begin{array}{l}17 \\
25 \\
42 \\
16\end{array}$ & $>0.05^{*}$ \\
\hline
\end{tabular}

*Estadísticamente significativo $(p<0.05)$ mediante $U$ de Mann-Whitney.

estrato medio alto, $28 \%$ a estrato medio y $14 \%$ a medio bajo. Estos resultados no mostraron diferencias estadísticamente significativas entre las adolescentes que habían tenido un END y las que no habían tenido ningún embarazo.

En relación con la escala de funcionalidad familiar de APGAR, $40.7 \%$ de las adolescentes que habían presentado un END procedían de familias con disfuncionalidad moderada, $25.9 \%$ tenía familias con disfuncionalidad severa, $22.2 \%$ disfuncionalidad leve y $11.1 \%$ venía de familias funcionales. De las estudiantes que no habían tenido ningún embarazo, $42 \%$ procedía de familias con disfunción moderada, $25 \%$ disfunción leve, $16 \%$ disfunción severa y $17 \%$ funcionales. Estos resultados no fueron estadísticamente significativos (Tabla 1).

Las adolescentes que habían tenido un END mantuvieron su primera relación sexual a la edad de 18.11 \pm 1.45 años y las que no habían tenido ningún embarazo a los $19.22 \pm 228$ años, con resultados estadísticamente significativos. La media de edad de las adolescentes cuando se quedaron embarazadas fue de $20.41 \pm 2.18$.

Aproximadamente $25 \%$ de las adolescentes, tanto de las que habían tenido un END como de las que no, indicó que su primera relación sexual no había sido voluntaria.
Todas las adolescentes que habían presentado END y $91 \%$ sin antecedente de embarazo tuvieron su primera relación sexual con su novio.

En cuanto al uso de métodos anticonceptivos, $59.3 \%$ de las adolescentes que habían tenido un END y $32 \%$ sin este antecedente indicaron no haber utilizado métodos anticonceptivos en su primera relación sexual, aspecto que mostró diferencias estadísticamente significativas (Tabla 2).

Los motivos de las mujeres con END para no usar anticonceptivos en su primera relación sexual fueron que la pareja no lo deseaba (36.8\%), la creencia de no quedar embarazada ( $26.3 \%$ ) y el desconocimiento (21.1\%). Las mujeres que no habían tenido embarazo señalaron como causas que la pareja no lo deseaba $(45,3 \%)$, desconocimiento (17\%) y la creencia de no quedar embarazada (17\%). No se identificaron diferencias estadísticamente significativas.

\section{Discusión}

En las universidades del Azuay que ofrecen la carrera de medicina, de 127 mujeres participantes, la frecuencia de END fue de $21.2 \%$. Estas cifras son similares a las descritas en 2014 por el Fondo de las Naciones Unidas para la Infancia, con datos de $20 \%$ de jóvenes 
Tabla 2. Comparación de las características sociodemográficas de las mujeres que han tenido o no embarazo

\begin{tabular}{|c|c|c|c|c|c|}
\hline \multirow[t]{3}{*}{ Variables } & \multicolumn{4}{|c|}{ Embarazo } & \multirow[t]{3}{*}{$\mathrm{p}$} \\
\hline & \multicolumn{2}{|c|}{ Sí } & \multicolumn{2}{|c|}{ No } & \\
\hline & $\mathrm{n}$ & Media \pm DE & $n$ & Media \pm DE & \\
\hline Edad primera relación & 27 & $18.11 \pm 1.45$ & 100 & $19.22 \pm 2.28$ & $<0.01^{*}$ \\
\hline Edad primera pareja & 27 & $19.78 \pm 3.40$ & 100 & $21.87 \pm 3.04$ & $<0.001^{*}$ \\
\hline \multirow[t]{2}{*}{ Edad del embarazo } & 27 & $20.41 \pm 2.18$ & & & \\
\hline & $n$ & $\%$ & $\mathrm{n}$ & $\%$ & \\
\hline $\begin{array}{l}\text { Voluntariedad en primera relación } \\
\text { Voluntaria } \\
\text { No voluntaria }\end{array}$ & $\begin{array}{l}20 \\
7\end{array}$ & $\begin{array}{l}74.1 \\
25.9\end{array}$ & $\begin{array}{l}75 \\
25\end{array}$ & $\begin{array}{l}75 \\
25\end{array}$ & $>0.05^{\star}$ \\
\hline $\begin{array}{l}\text { Relación con primera pareja } \\
\text { Amigo, vecino, conocido } \\
\text { Novio } \\
\text { Esposo/conviviente }\end{array}$ & $\begin{array}{c}0 \\
27 \\
0\end{array}$ & $\begin{array}{c}0 \\
100 \\
0\end{array}$ & $\begin{array}{c}6 \\
91 \\
3\end{array}$ & $\begin{array}{c}6 \\
91 \\
3\end{array}$ & $>0.05^{\star}$ \\
\hline $\begin{array}{l}\text { Anticonceptivos primera relación } \\
\text { Sí } \\
\text { No }\end{array}$ & $\begin{array}{l}11 \\
16\end{array}$ & $\begin{array}{l}40.7 \\
59.3\end{array}$ & $\begin{array}{l}68 \\
32\end{array}$ & $\begin{array}{l}68 \\
32\end{array}$ & $<0.01$ \\
\hline
\end{tabular}

embarazadas en América Latina y el Caribe; ${ }^{2}$ así como a las encontradas en 2013 por Zhou en estudiantes chinas, donde la prevalencia fue de $19.8 \%{ }^{33}$ y por Morales, en Cuba, con prevalencia de $18.7 \% .^{34}$

Por el contrario, nuestros resultados difieren de otros que muestran cifras más altas, como el estudio de Shu en China en 2016, en el que se registró $34.03 \%,{ }^{35}$ o el realizado por Ariza, en 2014, en jóvenes colombianas, que mostró cifras de END de $32.3 \%$; $^{10}$ en 2015, Wang encontró $31.8 \%$ en China. ${ }^{36}$ En otro trabajo realizado en 2014 en España por Yago, la prevalencia de END fue de $29.8 \%$. $^{37}$

Otros estudios, como el realizado por Xiao Ming (2013) en China, mostraron datos de END inferiores a los obtenidos en el nuestro (15.1\%):38 Coronado, en una investigación realizada en España indicó $1 \%$ en estudiantes de medicina. ${ }^{39}$

El $18.5 \%$ de las mujeres en nuestro estudio que indicaron antecedente de embarazo había abortado. Estos datos son similares a los presentados por Scott (2017): $18 \%$ de las adolescentes que quedaban embarazadas también abortaba. ${ }^{12}$ En 2016, Shu indicó un escenario muy diferente: $96.7 \%$ de las adolescentes en esta situación abortaba. ${ }^{33}$ En ese mismo año, Coronado registró que abortaba $40 \%$ de las adolescentes estudiantes de medicina que quedaban embarazadas. ${ }^{39}$

Las adolescentes que habían tenido un END presentaron un estrato socioeconómico medio alto y medio (55.6 y $29.6 \%$, respectivamente). Estos resultados difieren de los obtenidos por Blanco en 2015, en
Venezuela, en los que la pobreza extrema y la no extrema fueron factores de riesgo para END. ${ }^{40}$ El estudio llevado a cabo por Scott en 2017, en Francia y Gran Bretaña, mostró que en las adolescentes de los estratos socioeconómicos alto y medio prácticamente no se producían END. ${ }^{12}$

En nuestro estudio, las adolescentes que habían tenido un END procedían mayoritariamente de familias con disfunción moderada. Resultados similares fueron los presentados por Soto en un estudio en México, donde la funcionalidad familiar fue normal en las adolescentes con END. ${ }^{20}$ Los hallazgos anteriores difieren de los de Blanco en Venezuela, quien relacionó el embarazo con disfuncionalidad severa. ${ }^{40}$ Los resultados de Ariza y Betancur, en Colombia, indican que la familia disfuncional o conflictiva es un factor de riesgo para END. ${ }^{10,18} \mathrm{En}$ 2013, Aquino publicó un estudio sobre adolescentes ecuatorianas que revelaba que los problemas familiares y el maltrato familiar predisponían a END. ${ }^{19}$

La edad promedio de la primera relación sexual entre las estudiantes que presentaron END fue de 18.11 \pm 1.45 años, que coincide con los resultados referidos por Zhou en China, que muestran que a menor edad del debut sexual, más riesgo de END. ${ }^{33,35}$ Okigbo y Panova, en 2015 y 2016, encontraron relación entre END y edad de debut sexual menor a 15 años..$^{41,42}$

El $59.3 \%$ de las adolescentes de nuestro estudio que habían tenido un END no había utilizado anticonceptivos en la primera relación. Estos datos coinciden con los presentados en los estudios de Zhou y 
Shu. ${ }^{33,35}$ El $100 \%$ de embarazadas en un estudio llevado a cabo en adolescentes en México reconoció la falta de uso de anticonceptivos en su primera relación sexual como causa de su END. ${ }^{43}$

En conclusión, un porcentaje importante de las adolescentes de nuestro estudio, a pesar de ser jóvenes universitarias de medicina, proceder de familias con un grado de funcionalidad moderado y con nivel socioeconómico medio alto había tenido algún embarazo no deseado a temprana edad.

\section{Agradecimientos}

Nuestro más sincero agradecimiento a las jóvenes de la Universidad de Cuenca, Universidad Católica de Cuenca y Universidad del Azuay, por su incondicional colaboración, la cual hizo posible la realización de este estudio.

\section{Bibliografía}

1. Syme M. The evolving concept of older adult sexual behavior and its benefits. Generations. 2014:38:35-41.

2. Fondo de las Naciones Unidas para la Infancia. Vivencias y relatos sobre el embarazo en adolescentes. Una aproximación a los factores culturales, sociales y emocionales a partir de un estudio en seis países de la región. Informe final. Panamá: UNICEF; 2014.

3. Saeteros-Hernández RC, Pérez-Piñero CJ, Sanabria-Ramos CG. Vivencias de la sexualidad en estudiantes universitarios. Rev Cuba Salud Publica. 2013:39:915-928.

4. Núñez-Castillo J, Derluyn I, Valcke M. Young people's expressed needs for comprehensive sexuality education in Ecuadorian schools. Sex Educ. 2018:18:14-31

5. Jerves E, López S, Castro C, Ortiz W, Palacios M, Rober P, et al. Understanding parental views of adolescent sexuality and sex education in Ecuador: a qualitative study. Sex Educ. 2014;14:14-27.

6. Society for Adolescent Health and Medicine. Improving knowledge about, access to, and utilization of long-acting reversible contraception among adolescents and young adults. J Adolesc Health. 2017;60:472-474.

7. Corona HF, Funes DF. Abordaje de la sexualidad en la adolescencia. Rev Med Clin Las Condes. 2015;26:74-80.

8. Mendes N, Palma F, Serrano F. Sexual and reproductive health of Portuguese adolescents. Int J Adolesc Med Health. 2014;26:3-12.

9. United Nations Population Foundation [sitio web]. State of World Population 2017 EE. UU.: United Nations Population Foundation; 2017.

10. Ariza-Riaño NE, Sanabria MLV, Díaz JMO. Caracterización del embarazo adolescente en dos ciudades de Boyacá, Colombia. Rev Virtual Univ Catolica Norte. 2014;42:154-63.

11. Arias RM, Arias NA. Maternidad adolescente, desigualdad social y exclusión educativa en el Norte de Santander (Colombia) y Táchira (Venezuela). Espac Abierto Cuad Venez Sociol. 2017;26:121-138.

12. Scott RH, Bajos N, Slaymaker E, Wellings $\mathrm{K}$, Mercer $\mathrm{CH}$. Understanding differences in conception and abortion rates among under-20 year olds in Britain and France: examining the contribution of social disadvantage. PloS One. 2017;12:e0186412.

13. Ministerio de Salud Pública del Ecuador. Plan Nacional de Salud Sexual y Salud Reproductiva 2017-2021. Ecuador: Ministerio de Salud Pública del Ecuador; 2017.

14. Rodríguez-Vignoli J. La reproducción en la adolescencia y sus desigualdades en América Latina. Introducción al análisis demográfico, con énfasis en el uso de microdatos censales de la ronda de 2010. Chile: Comisión Económica para América Latina y el Caribe; 2014.

15. Burneo-Salazar C, Córdova-Páez A, Gutiérrez MJ, Ordóñez A. Embarazo adolescente en el marco de la Estrategia Nacional Intersectorial de Planificación Familiar (ENIPLA) 2014 y el Plan Nacional de Fortalecimiento de la Familia 2015 [sitio web]. Ecuador: Montecristi Vive; 2015.

16. Adebayo AM, Ajuonu EJ, Betiku BO. Family functionality and parenta characteristics as determinants of sexual decision-making of in-school youths in a semi-urban area of Southwest Nigeria. Int J Adolesc Med Health. 2016;28:413-418.
17. Canales J. Padres tóxicos. Legado disfuncional de una infancia. México: Paidós; 2014

18. Rojas-Betancur M, Méndez-Villamizar R, Álvarez-Nieto C. El papel de la familia en la normalización del embarazo a temprana edad. Rev Encuentros. 2016;14:139-150.

19. Aquino R, Jacqueline V. Incidencia del embarazo en la adolescencia en mujeres de 12 a 18 años en Maternidad Mariana de Jesús de septiembre 2012 a febrero del 2013. [Tesis de pregrado]. Ecuador: Universidad de Guayaquil; 2013.

20. Soto-Acevedo F, Alberto León-Corrales I, Castañeda-Sánchez O. Calidad de vida y funcionalidad familiar en adolescentes embarazadas derechohabientes de una unidad de medicina familiar del estado de Sonora, México. Aten Fam. 2015;22:64-67.

21. Baiz JB, Morales FM, Pereyra LP. Aspectos psicosociales de la salud sexual y reproductiva en estudiantes universitarios. Salud Soc. 2016;7:180-195.

22. Córdova-Pozo K, Chandra-Mouli V, Decat P, Nelson E, De Meyer S, Jaruseviciene $\mathrm{L}$, et al. Improving adolescent sexual and reproductive health in Latin America: reflections from an International Congress. Reprod Health. 2015;12:11.

23. González-Rugel E, Ortega-Mendoza WG. Percepciones sobre maternidad y paternidad en adolescentes hombres y mujeres, el caso de cuatro colegios del cantón Cuenca, período 2009-2010. [Tesis de pregrado]. Ecuador: Universidad de Cuenca; 2013.

24. Sam-Soto $S$, Osorio-Caballero $M$, Rodríguez-Guerrero $R$, Pérez-Ramírez N. Comportamiento sexual y anticoncepción en la adolescencia. Acta Pediatr Mex. 2014;35:490-498.

25. De Looze $M$, Constantine NA, Jerman $P$, Vermeulen-Smit $E$, Bogt $T$. Parent-adolescent sexual communication and its association with adolescent sexual behaviors: a nationally representative analysis in the Netherlands. J Sex Res. 2015;52:257-268.

26. Yaphe J. Is the family APGAR dead? A reappraisal of family assessment tools in Portugal. Rev Port Med Geral E Fam. 2013;29:14-15.

27. Cuba-Suárez AM, Alcalá-Espinoza M. APGAR familiar: una herramienta para detectar disfunción familiar. Rev Med La Paz. 2014;20:53-57.

28. Gómez-Clavelina FJ, Ponce-Rosas ER. Una nueva propuesta para la interpretación de Family APGAR (versión en español). Aten Fam. 2010; 17:102-106.

29. Benítez-Mesa M, Dunia-Dahdah MR, Rodríguez-Morales AJ. Evaluación del método de estratificación social Graffar-Méndez Castellano. 2011.

30. Cardozo Castellano de A RA, Poveda de Agustín JM, Guevara Rivas H, Ortunio Calabrés M, Loaiza Borges L, Torres San Narciso E. Características socio-demográficas de estudiantes de Medicina de dos Universidades Públicas en España y en Venezuela. 2009-2010. Vitae. 2013:54:1-5

31. Millán A, D'Aubeterre ME, Calvanese N. Propiedades psicométricas de la escala de preferencias vespertinidad/matutinidad en una muestra multiocupacional de trabajadores venezolanos. Rev Cuba Salud Trab. 2012:13:37-49.

32. Mendoza-Pinto C, Méndez-Martínez S, Soto-Santillán P, Galindo-Herrera J, Pérez-Contreras I, Macías-Díaz S, et al. Socioeconomic status and organ damage in Mexican systemic lupus erythematosus women. Lupus. 2015;24:1227-1232.

33. Zhou Y, Xiong C, Xiong J, Shang X, Liu G, Zhang M, et al. A blind area of family planning services in China: unintended pregnancy among unmarried graduate students. BMC Public Health. 2013:13:198.

34. Morales-Díaz E, Rojas S, María A, González M, Roberto S, Miranda-Gómez $O$. Embarazo no deseado en alumnas universitarias. Rev Cuba Med Mil. 2013;42:153-163.

35. Shu C, Fu A, Lu J, Yin M, Chen Y, Qin T, et al. Association between age at first sexual intercourse and knowledge, attitudes and practices regarding reproductive health and unplanned pregnancy: a cross-sectional study. Public Health. 2016;135:104-113.

36. Wang $\mathrm{H}$, Long L, Cai H, Wu Y, Xu J, Shu C, et al. Contraception and unintended pregnancy among unmarried female university students: a cross-sectional study from China. PLoS One. 2015;10:e0130212.

37. Yago-Simón T, Tomás-Aznar C. Variables sociodemográficas relacionadas con embarazos no planificados en jóvenes de 13 a 24 años. Rev Esp Salud Publica. 2014:88:395-406

38. Yu XM, Guo SJ, Sun YY. Sexual behaviours and associated risks in Chinese young people: a meta-analysis. Sex Health. 2013:10:424-433.

39. Coronado-Martin P, Delgado-Miguel C, Rey-Cañas A, A. Herráiz M. Sexual and reproductive health in Spanish university students. A comparison between medical and law students. 2017;11:97-101.

40. Blanco L, Cedre O, Guerra ME. Factores determinantes del embarazo adolescente. Odontol Pediatr Lima. 2015;14(2):109-119.

41. Okigbo CC, Speizer IS. Determinants of sexual activity and pregnancy among unmarried young women in urban Kenya: a cross-sectional study. PLoS One. 2015:10:1-17.

42. Panova OV, Kulikov AM, Berchtold A, Suris JC. Factors associated with unwanted pregnancy among adolescents in Russia. J Pediatr Adolesc Gynecol. 2016;29:501-505

43. Otero LMR. El embarazo a temprana edad. Perspectiva de progenitores y madres adolescentes. Rev Sobre Infancia Adolesc. 2016;10:81-107. 